\title{
About the manuscript Arica et al
}

\author{
Balahan Makay
}

Received: 25 November 2011/ Accepted: 4 March 2012/Published online: 16 March 2012

(C) Springer-Verlag 2012

Dear Editor,

I read the article by Arıca et al. entitled "Evaluation of mean platelet volume in children with FMF" with interest. The authors cited one of our studies in reference number 17 (Makay B, Türkyılmaz Z, Unsal E. Mean platelet volume in children with FMF. Clin Rheumatol 2009;28:975-978). In the discussion part, in paragraph 3, they stated that "MPV values of attack-free FMF patients were found surprisingly less compared to those of healthy individuals." However, this is not our result. We found that patients in acute attack had significantly lower MPV values than both healthy controls and attack-free patients. Besides, there was no difference between attack-free patients and healthy controls regarding the MPV. We kindly ask your help to correct this mistake.

I also noticed that reference numbers are not in correct order. Reference numbers of 15, 16, and 17 should be checked out. Reference 15 corresponds to "Çoban et al." and Ref. 16 corresponds to "Makay et al." in the last paragraph of introduction. However, ref. 15 becomes "Tunca et al." in the first paragraph of discussion, and the other abovementioned references become 16, 17, respectively. Thank you for your help in advance. 